\title{
Efficacy and short-term side effects of sitagliptin, vildagliptin and saxagliptin in Chinese diabetes: a randomized clinical trial
}

\author{
Xiao-jun Zhou ${ }^{1, *}$, Lin Ding ${ }^{1, *}$, Jia-xin Liu' ${ }^{1}$, Le-qun Su${ }^{2}$, Jian-jun Dong ${ }^{3}$ and Lin Liao ${ }^{1}$ \\ 'Department of Endocrinology, Shandong Provincial Qianfoshan Hospital, Shandong University, Jinan, Shandong, China \\ 2Department of Pharmacy, Shandong Provincial Qianfoshan Hospital, Shandong University, Jinan, Shandong, China \\ ${ }^{3}$ Division of Endocrinology, Department of Medicine, Qilu Hospital of Shandong University, Shandong University, Jinan, Shandong, China
}

Correspondence should be addressed to L Su or J Dong or L Liao: slequn@126.com or cwc_Il@sdu.edu.cn or liaolin@sdu.edu.cn

*(X Zhou and L Ding contributed equally to this work)

\begin{abstract}
Aims: To investigate the difference in the efficacy among dipeptidyl peptidase-4 (DPP-4) inhibitors in Chinese adults with newly diagnosed diabetes.

Materials and methods: In a multicenter, randomized study, we enrolled adults who were either treatment naive or off prior anti-hyperglycemic therapy for at least 3 months. Eligible patients had hemoglobin A1c (HbA1c) concentrations of 6.5-9.5\%. Three hundred patients had been randomly allocated to sitagliptin $100 \mathrm{mg}$, once daily; vildagliptin $50 \mathrm{mg}$, twice daily and saxagliptin $5 \mathrm{mg}$, once daily for 12 weeks. Patients and investigators were masked to treatment assignment. The primary endpoint was change from baseline in $\mathrm{HbA} 1 \mathrm{c}$ at week 12. This study was completed and registered with ClinicalTrials.gov, number NCT 01703637.

Results: Totally 277 patients were enrolled in the final analysis, and 93 patients received sitagliptin, 94 received vildagliptin and 90 received saxagliptin. Compared with baseline, adjusted mean differences in change from baseline $\mathrm{HbA} 1 \mathrm{c}$ at week 12 were $-0.50 \%$ ( $95 \% \mathrm{Cl}:-0.20$ to -0.90$),-0.65 \%$ ( $95 \% \mathrm{Cl}:-0.40$ to -1.40$),-0.70$ (95\% Cl: -0.50 to -1.00$)$ for sitagliptin, vildagliptin and saxagliptin group, respectively. The overall HbA1c-lowering effect was similar for all three selected DPP-4 inhibitors after adjustment for age and baseline $\mathrm{HbA} 1 \mathrm{c}$. Notably, in secondary outcome analysis, patients in vildagliptin group showed a significant decrease in total cholesterol levels, compared with participants in sitagliptin and saxagliptin groups. No significant between-group difference was shown in adverse events (AE).

Conclusions: The overall HbA1c-lowering effect and incidence of AE were similar for sitagliptin, vildagliptin and saxagliptin in Chinese adults with newly diagnosed diabetes.
\end{abstract}
Key Words
- dipeptidyl peptidase-4 inhibitors
- hemoglobin A1c
- Chinese
- diabetes

Endocrine Connections (2019) 8, 1-8

\section{Introduction}

Diabetes, as one of the largest global health emergencies in the 21st century, has increased with a burgeoning trend. The latest International Diabetes Federation (IDF) Diabetes Atlas indicated that in 2016 the estimated number of people with diabetes worldwide has reached 425 million (1). Particularly, China has the world's largest number of adults with diabetes, and China is on the top two countries for diabetes-related health expenditure, with 110 billion US dollars in 2017 (1). In addition, the recent report from a nationally representative cross-sectional survey in mainland China, reported a prevalence of $10.9 \%$ for diabetes, and $35.7 \%$ for prediabetes among Chinese

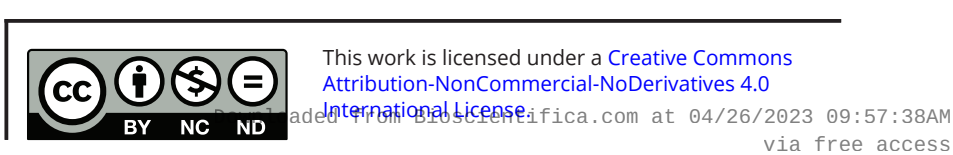


adults in 2013 (2). With the alarming disease burden of diabetes, the effective hypoglycemic therapy is pivotal. However, despite over 40 new treatment options for type 2 diabetes having been approved by the US Food and Drug Administration (FDA) since 2005, the proportion of patients with adequate glycemic control remains around $50 \%$ in the United States (3) and $39.7 \%$ in China (4).

Dipeptidyl peptidase-4 (DPP-4) inhibitors, characterized by low propensity of hypoglycemia and weight neutrality, have gradually become an important class in the armamentarium of oral glucose-lowering agents $(5,6)$. Currently, the consensus does not recommend DDP-4 inhibitors as the first line of treatment or as monotherapy on management of hyperglycemia in type 2 diabetes which has been reached by the American Diabetes Association (ADA) and the European Association for the Study of Diabetes (EASD) (7). Since sitagliptin, the first DPP-4 inhibitor, launched in 2006, 12 drugs have been available in the market of DPP-4 inhibitors, and by the year 2012, three DPP-4 inhibitors were available in China. So, we initiated the head-to-head study of the three DPP-4 inhibitors (sitagliptin, saxagliptin and vildagliptin).

Over a decade, much data have been accumulated from clinical trials and post-marketing studies of the DPP-4 inhibitors. Most current clinical trials focused on evaluating the combination of DPP-4 inhibitors with metformin and other hypoglycemic therapy, or monotherapy with DPP-4 inhibitors with placebo, rather than comparing within the class of DPP-4 inhibitors as the initial single therapy.

In addition, Cardiovascular (CV) Outcomes Trials showed that long-term safety studies on sitagliptin were neutral, whereas CV outcome trials on saxagliptin were associated with a significant increase in the rate of hospitalization for heart failure (SAVOR-TIMI 53), which was saxagliptin treatment only $(8,9)$. No long-term CV outcome trials safety data are available on vildagliptin. There is still scarcity of comparison between various DPP-4 inhibitors in the short-term side effects. In clinical practice, the selection of DPP-4 inhibitors is mainly based on physician's habit, and there is limited evidence that help professionals in choosing DPP-4 inhibitors (10). Cost and availability aside, sitagliptin and saxagliptin might be likely to be easily prescribed with one available dose, a once-daily dosing regimen, compared with vildagliptin. There is a paucity of direct head-to-head studies of DPP-4 inhibitors and most comparisons are indirect and the potential for glycemic improvement might not be uniform within the class. In addition, ethnic difference may exist when comparing the effectiveness of DPP-4 inhibitors among patients in various regions. Previous study indicated that DPP-4 inhibitors exhibit a better glucose-lowering efficacy in Asians than in other ethnic groups (11). However, there exists few evidence on the effectiveness of DPP-4 inhibitors in Asia. Therefore, we explored the therapeutic equivalence of saxagliptin, sitagliptin and vildagliptin as monotherapy among patients who were either treatment-naive or off prior anti-hyperglycemic agent therapy for at least 3 months, highlighting the differences and similarities between members of the DPP-4 inhibitors.

\section{Methods}

\section{Study design}

This is a 12-week, multicenter, randomized, double-blind comparative study in patients with newly diagnosed diabetes or off prior anti-hyperglycemic agent therapy for at least 3 months who had inadequate glycemic control undertaken in 16 hospitals in Shandong Province, China from October 2012 to January 2016. All three gliptins in this study were used at their expected maximal effective and recommended doses (in accordance with product labeling) for patients with newly diagnosed diabetes. Patients had been randomized to sitagliptin (Merck Sharp \& Dohme Italia SPA) $100 \mathrm{mg}$, once daily; vildagliptin (Novartis Pharma Stein AG) $50 \mathrm{mg}$, twice daily; and saxagliptin (AstraZeneca Pharmaceuticals LP) $5 \mathrm{mg}$, once daily for 12 weeks. The purpose of this study is to explore the differences in efficacy and short-term side effects of sitagliptin, vildagliptin and saxagliptin and to find which one is better in treating type 2 diabetes mellitus. The study was undertaken in accordance with the Declaration of Helsinki and the Guidelines for Good Clinical Practice. The Institutional Review Board of Shandong University approved the study protocol, and written informed consent was obtained from all participants.

In order to be strictly double-blinded, we performed randomization within each center. Besides, considering the different appearances of the three gliptins, placebo tablets (Qilu Pharmaceutical Co., Ltd, China) were used for blinding. We used three kinds of placebos to represent sitagliptin, vildagliptin and saxagliptin, accordingly. All patients in different drug groups received 3 pills in the morning (among which one is the true drug, the other two are placebos) and 1 pill in the evening (among which only participants in the vildagliptin group received the real drug pill, and others received placebo). 


\section{Study population}

Patients were recruited from 16 hospitals in Shandong Province, China. Eligible patients were male or female, 18-78 years of age and had type 2 diabetes with hemoglobin A1c (HbA1c) values ranging between 6.5 and $9.5 \%$. Women of childbearing potential were required to have a negative urine pregnancy test and agreed to use adequate contraception throughout the study and for up to 4 weeks after completion. All participants signed the written informed consent.

Patients were excluded if they had one or more of the following criteria:

1. patients in pregnancy or lactation period;

2. ever received any kind of oral hypoglycemic drug or insulin in the recent 3 months;

3. patients were currently receiving treatment with a cytochrome P450 3A4 inducer or depressor, a systemic corticosteroid or a human immunodeficiency virus anti-viral medication;

4. gastrointestinal surgery that could affect drug absorption;

5. patients with hemoglobinopathy or rapidly progressing renal disease or autoimmune skin disorder;

6. a recent history of alcohol or drug abuse within the past 12 months;

7. any contraindication listed in the package inserts of the study drugs;

8. a history of or currently having acute or chronic pancreatitis;

9. type 1 diabetes; a history of diabetic ketoacidosis or hyperosmolar nonketonic coma;

10. New York Heart Association class III or IV congestive heart failure; left ventricular ejection fraction $\leq 40 \%$; a major cardiovascular event within the past 6 months;

11. significant abnormal liver function, defined as aspartate aminotransferase (AST) or alanine aminotransferase (ALT) $>2$ times the upper limit of normal or total bilirubin $>34 \mu \mathrm{mol} / \mathrm{L}$ ( $>2 \mathrm{mg} / \mathrm{dL}$ ) or a history of positive serologic evidence of infectious liver disease;

12. clinical signs or symptoms of active liver disease and/or significant abnormal liver function;

13. patients with any clinically significant abnormality identified on physical examination, electrocardiogram (ECG) or laboratory tests that, in the judgment of the investigator, would compromise the patients' safety or successful participation in the clinical study;

14. fasting plasma glucose level $>13.3 \mathrm{mmol} / \mathrm{L}$;

15. creatinine clearance rate $\leq 90 \mathrm{~mL} / \mathrm{min}$.

\section{Outcomes}

\section{Primary outcome measures}

1. Change from baseline in HbA1c levels at week 12.

2. Change from baseline reflects the week 12 value minus the baseline value. HbA1c represents the percentage of glycosylated hemoglobin.

\section{Secondary outcome measures}

1. Change from baseline in fasting plasma glucose (FPG) at week 12 .

2. Change from baseline in 2-h postprandial glucose (PPG) at week 12.

3. Change from baseline in body weight at week 12 .

4. Change from baseline in fasting plasma lipids at week 12 .

5. Fasting plasma lipid parameters include total cholesterol (TC), high-density lipoprotein cholesterol (HDL-C) and low-density lipoprotein cholesterol (LDL-C).

6. The proportion of patients achieving $\mathrm{A} 1 \mathrm{C}<7 \%$ and achieving $\mathrm{A} 1 \mathrm{C}<6.5 \%$ at week 12 .

\section{Procedure}

HbA1c was recorded at baseline (week 0), week 6 and week 12. All blood samples were shipped in dry ice to and then tested in the central laboratory in Qianfoshan Hospital, Jinan, Shangdong. Venous blood samples were obtained from the patients for laboratory tests after at least $8 \mathrm{~h}$ of overnight fasting. Oral glucose tolerance test (OGTT) with a $75 \mathrm{~g}$ glucose load was performed. Blood glucose from the fasting and 2-h postloading blood sampling was measured using the glucose oxidase method on an autoanalyzer (Cobas 8000; Roche). HbA1c was detected by HPLC (BIO-RAD D-10). Measurement of fasting serum TC, LDL-C, HDL-C and fasting serum alanine aminotransferase, creatine $(\mathrm{Cr})$ and total bilirubin from fasting plasma samples was undertaken on the autoanalyzer (Cobas 8000; Roche). Blood cell analysis was performed using whole blood automatic analyzer (SYSMEX xs900i, Kobe, Japan).

\section{Statistical analyses}

SAS version 9.4 was used for all analyses. Data are expressed as mean (s.D.), median (Q1-Q3), estimates (SE) or differences with 95\% CI. Efficacy analysis included all participants randomly assigned to treatment groups who had received at least one dose of study drug and had at 
least one on-treatment assessment of any primary or secondary efficacy variable. The adjusted mean changes from baseline to endpoint (with last observation carried forward) were compared between treatments using an ANCOVA model, and adjusted for age, as age is significantly different at baseline among the three gliptin groups. Efficacy data were censored at the start of rescue medication. $P<0.05$ was considered to be statistically significant. This trial is registered with ClinicalTrials.gov, number, NCT01703637.

\section{Results}

\section{Demographic characteristics of study participants at baseline}

A total of 300 patients with type 2 diabetes were randomized, and 277 patients comprised the full analysis set. Seven of 100 subjects withdrew from sitagliptin, 6 of 100 from vildagliptin and 10 of 100 from saxagliptin treatment. The withdrawal rates were not significantly different between the groups (Supplementary Fig. 1, see section on supplementary data given at the end of this article). According to treatment group, 93 patients were treated with sitagliptin (100 mg once daily), 94 patients with vildagliptin (50 mg twice daily) and 90 patients with saxagliptin ( $5 \mathrm{mg}$ once daily). There were 36 patients with a history of oral hypoglycemic drug use, and 241 patients were truly drug naïve among the 277 participants. Table 1 summarizes the demographic characteristics of study participants at baseline according to the three DPP-4 inhibitors. There were no significant differences in clinical characteristics among the randomized population, except age. Participants in the sitagliptin group are elder than participants in the vidagliptin or saxagliptin groups $(P$ value $=0.02)$. Besides, BMI, lipid profiles, blood glucose levels, liver enzymes and creatinine were similar among three DPP-4 inhibitor groups after adjusting for age.

\section{Demographic characteristics of study participants at week 12}

The biochemical characteristics among participants after 12-week treatment period are represented in Supplementary Table 1. After adjusting age and baseline HbA1c level, all biochemical variables including BMI, lipid profile, blood glucose levels, liver enzymes and creatinine showed no statistical difference among patients.

\section{Primary efficacy measurements from baseline to week 12}

The age and baseline HbA1c-adjusted mean levels of HbA1c, FPG and PPG from baseline to week 12, as well as changes of these glucose indicators and percentages of patients achieving a target $\mathrm{HbA} 1 \mathrm{c}<7 \%$ and $<6.5 \%$ during the 12 -week treatment period, are represented in Table 2. Mean HbA1c was $6.5 \%$ in both sitagliptin and vildagliptin groups, but $6.6 \%$ in the saxagliptin group. The changes of HbA1c from baseline to week 12 in sitagliptin, vildagliptin and saxagliptin groups were $0.50,0.65$ and $0.70 \%$, respectively. Both the levels and changes of HbA1c among the three DPP-4 inhibitor groups showed no significant differences after 12-week treatment. The mean FPG and

Table 1 Demographic characteristics of study participants at baseline. Data were means \pm S.D. or medians (interquartile ranges) for skewed variables or numbers (proportions) for categorical variables.

\begin{tabular}{|c|c|c|c|c|}
\hline & Sitagliptin $(N=93)$ & Vildagliptin $(N=94)$ & Saxagliptin $(N=90)$ & $P$ value \\
\hline Age (years) & $55.1 \pm 9.3$ & $49.3 \pm 10.0$ & $48.7 \pm 11.2$ & 0.02 \\
\hline $\mathrm{BMI}\left(\mathrm{kg} / \mathrm{m}^{2}\right)$ & $27.0 \pm 3.4$ & $26.8 \pm 3.1$ & $27.1 \pm 2.9$ & 0.93 \\
\hline $\mathrm{TC}(\mathrm{mmol} / \mathrm{L})$ & $5.1(4.4-5.6)$ & $5.0(4.2-5.5)$ & $5.1(4.6-6.0)$ & 0.74 \\
\hline LDL-C (mmol/L) & $3.3 \pm 0.8$ & $3.0 \pm 0.9$ & $3.3 \pm 0.9$ & 0.56 \\
\hline HDL-C (mmol/L) & $1.2 \pm 0.3$ & $1.3 \pm 0.5$ & $1.3 \pm 1.2$ & 0.87 \\
\hline FPG (mmol/L) & $8.3 \pm 1.6$ & $9.0 \pm 2.6$ & $8.4 \pm 1.6$ & 0.40 \\
\hline PPG (mmol/L) & $14.9 \pm 4.9$ & $16.1 \pm 5.5$ & $13.3 \pm 4.6$ & 0.17 \\
\hline HbA1c (\%) & $7.10(6.70-7.80)$ & $7.50(6.90-8.00)$ & $7.40(6.80-7.90)$ & 0.26 \\
\hline ALT (IU/L) & $22.1(16.0-31.5)$ & $20.0(17.1-32.0)$ & $24.0(19.2-38.0)$ & 0.11 \\
\hline AST (IU/L) & $18.0(15.0-23.0)$ & $17.0(14.4-22.0)$ & $20.0(16.0-26.0)$ & 0.68 \\
\hline $\mathrm{Cr}(\mu \mathrm{mol} / \mathrm{L})$ & $64.4 \pm 16.4$ & $67.61 \pm 17.0$ & $62.2 \pm 10.3$ & 0.41 \\
\hline $\mathrm{BIL}(\mu \mathrm{mol} / \mathrm{L})$ & $19.6 \pm 30.6$ & $14.2 \pm 6.5$ & $16.4 \pm 6.4$ & 0.61 \\
\hline WBC $\left(10^{9} / L\right)$ & $5.9 \pm 1.0$ & $6.5 \pm 1.4$ & $6.5 \pm 1.7$ & 0.24 \\
\hline
\end{tabular}

$P$ value was calculated for Chi-square test across the three groups.

BIL, total bilirubin; BMI, body mass index; Cr, creatine; FPG, fasting plasma glucose; HbA1c, hemoglobin A1c; HDL-C, high-density lipoprotein cholesterol; LDL-C, low-density lipoprotein cholesterol; TC, total cholesterol; WBC, white blood cell.

https://ec.bioscientifica.com https://doi.org/10.1530/EC-18-0523
(C) 2019 The authors Published by Bioscientifica Ltd

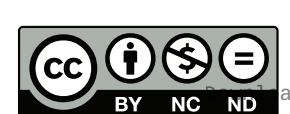

This work is licensed under a Creative Commons Attribution-NonCommercial-NoDerivatives 4.0 Internationab bicense.ifica com at 04/26/2023 $09: 57: 38 \mathrm{Am}$ 
Table 2 Primary efficacy measurements from baseline to week 12.

\begin{tabular}{|c|c|c|c|c|}
\hline & Sitagliptin group $(N=93)$ & Vildagliptin group $(N=94)$ & Saxagliptin group $(N=90)$ & $P *$ value \\
\hline \multicolumn{5}{|l|}{$\mathrm{HbA} 1 \mathrm{c}$ at week $12(\%)$} \\
\hline Baseline & $7.10(6.70-7.80)$ & $7.50(6.90-8.00)$ & $7.40(6.80-7.90)$ & \\
\hline Week 12 & $6.50(6.30-7.10)$ & $6.50(6.20-7.10)$ & $6.60(6.20-6.90)$ & \\
\hline Change from baseline & $0.50(0.20-0.90)$ & $0.65(0.40-1.40)$ & $0.70(0.50-1.00)$ & 0.18 \\
\hline \multicolumn{5}{|l|}{ FPG at week 12 (mmol/L) } \\
\hline Baseline & $8.3 \pm 1.6$ & $9.0 \pm 2.6$ & $8.4 \pm 1.6$ & \\
\hline Week 12 & $7.7 \pm 1.3$ & $8.1 \pm 2.7$ & $7.7 \pm 2.8$ & \\
\hline Change from baseline & $0.87 \pm 1.17$ & $1.24 \pm 3.76$ & $1.30 \pm 2.08$ & 0.78 \\
\hline \multicolumn{5}{|l|}{ PPG at week $12(\mathrm{mmol} / \mathrm{L})$} \\
\hline Baseline & $14.9 \pm 4.9$ & $16.1 \pm 5.5$ & $13.3 \pm 4.6$ & \\
\hline Week 12 & $13.3 \pm 4.8$ & $12.3 \pm 4.4$ & $11.6 \pm 4.6$ & \\
\hline Change from baseline & $2.1 \pm 3.2$ & $3.9 \pm 5.6$ & $2.1 \pm 5.3$ & 0.89 \\
\hline \multicolumn{5}{|c|}{ The number of patients achieving $\mathrm{A} 1 \mathrm{C}<7 \%$ at week 12} \\
\hline Baseline & 12 & 9 & 10 & \\
\hline Week 12 & 24 & 24 & 26 & 0.10 \\
\hline \multicolumn{5}{|c|}{ The number of patients achieving A1C <6.5\% at week 12} \\
\hline Week 12 & 15 & 14 & 11 & 0.74 \\
\hline
\end{tabular}

PPG showed similar trend among sitagliptin, vildagliptin and saxagliptin groups with no statistical changes after 12-week treatment. In addition, the percentages of patients achieving a target $\mathrm{HbA} 1 \mathrm{c}<7 \%$ during the 12 -week treatment period in sitagliptin, vildagliptin and saxagliptin groups were 24/93, 24/94 and 26/90, respectively (age-adjusted $P=0.69$ ), and the corresponding percentages of patients achieving a target HbA1c $<6.5 \%$ was 15/93, 14/94 and 11/90, respectively (age-adjusted $P=0.21$ ). Percentage of patients achieving an HbA1c target of $<7.0 \%$ or $<6.5 \%$ was similar in the three gliptin groups. Besides, HbA1c levels among sitagliptin, vildagliptin and saxagliptin groups from baseline to week 6 and week 12, also showed insignificant difference (Fig. 1).

\section{Secondary efficacy measurements from baseline to week 12}

Changes of biochemical variables including BMI, lipids, liver enzymes and creatinine were shown in Table 3 after 12 -week treatment. Notably, patients in vildagliptin group showed a significant decrease in TC levels, compared with participants in sitagliptin and saxagliptin. The changes of BMI, LDL-C, HDL-C, ALT, AST and Cr were not significant among sitagliptin, vildagliptin and saxagliptin groups (Table 3 ).

\section{Adverse events}

Adverse events, including fever, gastrointestinal discomfort and ocular hyperemia/streaming eyes were similar among the three study populations.
During 12-week study, one patient in sitagliptin group withdrew from the study - who discontinued due to asthma with unknown reasons (Table 4).

\section{Discussion}

This study is the first to directly compare the efficacy and short-term side effects of three DPP-4 inhibitors, namely sitagliptin, vildagliptin and saxagliptin, in patients with newly diagnosed diabetes or off prior anti-hyperglycemic agent therapy for at least 3 months who had inadequate glycemic control in 16 hospitals in Shandong Province, China. The overall HbA1c-lowering effect was similar for all three selected DPP-4 inhibitors.

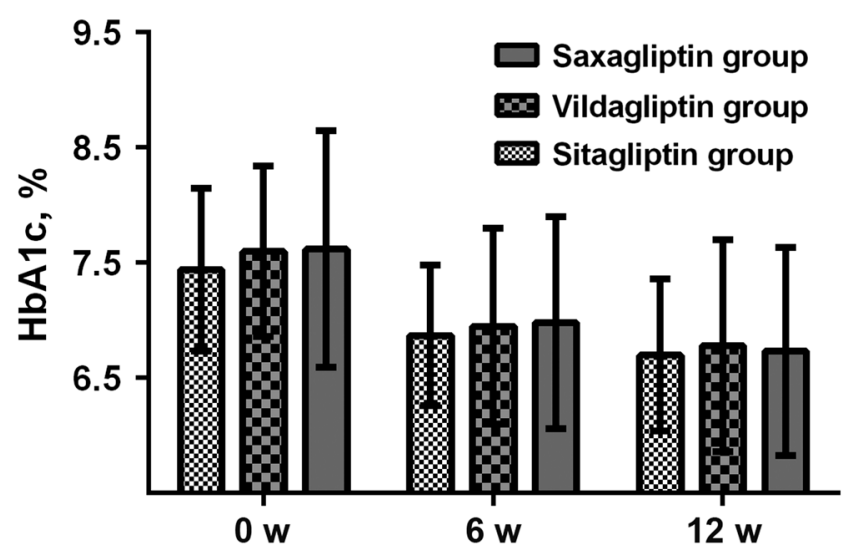

Figure 1

Hemoglobin A1c (HbA1c) levels among sitagliptin, vildagliptin and saxagliptin groups from baseline to week 12 . 
Table 3 Secondary efficacy measurements from baseline to week 12. Data were means \pm S.D. or medians (interquartile ranges) for skewed variables or numbers (proportions) for categorical variables.

\begin{tabular}{lcc}
\hline & & Sitagliptin $(N=93)$ \\
\cline { 1 - 1 } BMI $\left(\mathrm{kg} / \mathrm{m}^{2}\right)$ change & $-0.5 \pm 1.9$ \\
TC $(\mathbf{m m o l} / \mathrm{L})$ change & & $\mathbf{0 . 1}(-\mathbf{0 . 4}$ to $\mathbf{0 . 4})$ \\
LDL-C $(\mathrm{mmol} / \mathrm{L})$ change & & $-0.2 \pm 0.8$ \\
HDL-C $(\mathrm{mmol} / \mathrm{L})$ change & & $-0.00 \pm 0.2$ \\
ALT $(\mathrm{IU} / \mathrm{L})$ change & & $1.0(-1.0$ to 5.0$)$ \\
AST $(\mathrm{IU} / \mathrm{L})$ change & $-1.1 \pm 9.4$ \\
Cr $(\mu \mathrm{mol} / \mathrm{L})$ change & $8.1 \pm 34.0$ \\
BIL $(\mu \mathrm{mol} / \mathrm{L})$ change & $-0.2 \pm 2.0$ \\
WBC $\left(10^{9} / \mathrm{L}\right)$ change & \\
\hline
\end{tabular}

\begin{tabular}{c}
\hline Vildagliptin $(N=94)$ \\
$-0.1 \pm 0.7$ \\
$-\mathbf{0 . 1}(-0.5$ to 0.5$)$ \\
$-0.2 \pm 1.1$ \\
$0.06 \pm 0.4$ \\
$0.65(-7.3$ to 4.4$)$ \\
$-2.6(-8.1$ to 1.1$)$ \\
$2.3 \pm 11.7$ \\
$1.2 \pm 6.7$ \\
$-0.3 \pm 2.1$
\end{tabular}

\begin{tabular}{ccc}
\hline Saxagliptin $(N=90)$ & & $\boldsymbol{P *}$ value \\
\hline $0.3 \pm 0.8$ & & 0.41 \\
$\mathbf{0 . 5}(\mathbf{0 . 2}-\mathbf{1 . 0})$ & & $\mathbf{0 . 0 1}$ \\
$0.3 \pm 1.1$ & & 0.05 \\
$0.02 \pm 1.6$ & & 0.99 \\
$6.0(0.0-20.2)$ & & 0.08 \\
$1.5(-1.0$ to 6.2$)$ & & 0.42 \\
$-2.8 \pm 8.0$ & & 0.70 \\
$0.9 \pm 5.7$ & & 0.42 \\
$0.05 \pm 2.1$ & 0.62 \\
\hline
\end{tabular}

$P$ value was calculated for Chi-square test across the three groups. $P<0.05$ was considered to be statistically significant.

*Age and $\mathrm{HbA} 1 \mathrm{c}$-adjusted $P$ value.

BMI, body mass index; FPG, fasting plasma glucose; HbA1c, hemoglobin A1c; TC, total cholesterol; HDL-C, high-density lipoprotein cholesterol; LDL-C, low-density lipoprotein cholesterol; Cr, creatine; BIL, total bilirubin; WBC, white blood cell.

DPP-4 inhibitors have been shown to provide significant improvements in standard indices of glycemic control and are increasingly positioned earlier in treatment algorithms both as monotherapy and as add-on treatment $(12,13,14)$. The usage of DPP-4 inhibitor increased with time in both the United States and China (15). Although within the same class, sitagliptin, vildagliptin and saxagliptin have their own characteristics which could influence its effect and usage. Within the DPP-4 inhibitor class of medications, the choice of medication is a comprehensive assessment of patient characteristics, comorbid conditions, doctor's preference and insurance. In the US, sitagliptin was the most prescribed DPP-4 inhibitor, representing a $76.4 \%$ share in its class or equivalently 10.5 million prescriptions in the USA in 2012 , followed by saxagliptin (17.3\% share) and linagliptin (6.4\% share) (16). The latest drug trend report by Express Scripts reports that sitagliptin was among the top 10 drugs (and the only oral hypoglycemic) in the USA based on a per-member-per-year spending in 2016 (17). It has been reported that individuals who initiated saxagliptin demonstrated better adherence and were less likely to discontinue therapy compared to those that initiated linagliptin and sitagliptin (18). However, in full disclosure, each of these studies was supported by the manufacturer of saxagliptin.
Most previous study focused on sitagliptin given as monotherapy compared with a placebo or as an add-on therapy to oral hypoglycemic agents or insulin (14). There was no study directly comparing the hypoglycemic effect within those gliptins as monotherapy. Previous trials showed that, as add-on therapy in Chinese patients with type 2 diabetes, sitagliptin, vildagliptin and saxagliptin showed almost similar glycemic control and incidence of AE. However, for FPG control, saxagliptin demonstrated superiority to sitagliptin, while inferiority to vildagliptin (19). In addition, Tatosian et al. conducted a randomized, placebo-controlled, open-label, five-period crossover study reporting that inhibition of DPP-4 once-daily treatment with sitagliptin provided was significantly greater than saxagliptin or vildagliptin administered once daily, and similar to that provided by vildagliptin administered twice daily, through \%DPP-4 inhibition, derived by comparing DPP-4 activity $24 \mathrm{~h}$ (20). Unfortunately, antihyperglycemic efficacy was not fully evaluated in this study. Notably, the sitagliptin group had a numerically greater adjusted mean change from baseline in FPG than the saxagliptin group, although insignificantly (20). However, given as monotherapy, our study found that the three gliptins were almost equivalent in glucose control.

The HbA1c reductions seen with sitagliptin $100 \mathrm{mg}$, once daily were similar to the reductions observed with

Table 4 Adverse events.

\begin{tabular}{l}
\hline Side effects \\
\hline Fever \\
Ocular hyperemia, streaming eyes \\
Gastrointestinal discomfort \\
Total
\end{tabular}

\begin{tabular}{c}
\hline Sitagliptin $(N=93)$ \\
\hline 2 \\
1 \\
3 \\
6
\end{tabular}

\begin{tabular}{c}
\hline Vildagliptin $(N=94)$ \\
\hline 1 \\
0 \\
3 \\
4
\end{tabular}

\begin{tabular}{crr}
\hline Saxagliptin $(N=90)$ & & $P$ value \\
\cline { 1 - 1 } & & 0.55 \\
2 & 0.44 \\
4 & 0.98 \\
6 & 0.46 \\
\hline
\end{tabular}

Data are presented as number. Chi-square test was used for comparison of differences in the three groups.

https://ec.bioscientifica.com https://doi.org/10.1530/EC-18-0523 (c) 2019 The authors Published by Bioscientifica Ltd

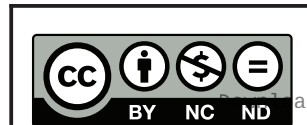

This work is licensed under a Creative Commons Attribution-NonCommercial-NoDerivatives 4.0 Internationab bicense.ifica com at 04/26/2023 $09: 57: 38 \mathrm{Am}$ 
vildagliptin $50 \mathrm{mg}$, twice daily, and saxagliptin $5 \mathrm{mg}$, once daily in patients with newly diagnosed diabetes. In our secondary endpoints, total cholesterol was significantly decreased in patients with vidagliptin treatment. DPP-4 has been noted as a novel adipokine potentially linking obesity to the metabolic syndrome (21). Higher visceral adipose tissue DPP4 gene expression in non-diabetic severely obese men with metabolic syndrome was observed than non-metabolic syndrome. An association between the DPP-4 methylation levels with plasma totalcholesterol levels in severe obesity was indicated, which suggests a link between the DPP-4 gene and plasma lipid metabolism (22), which might imply a potential mechanism for the finding of total cholesterol change in our study.

The present study has several strengths. Firstly, our study is the first randomized, multicenter study to comprehensively compare efficacy and short-term side effects of sitagliptin, vildagliptin and saxagliptin, in patients with newly diagnosed diabetes or off prior antihyperglycemic agent therapy for at least 3 months in Asia. Secondly, OGTT was performed among all participants and all three glycemic indexes for the diagnosis of diabetes - FPG, PPG and HbA1c concentrations - were obtained, which provide a comprehensive estimation of glycemic control. Thirdly, double-blinded method was strictly performed. Patients and investigators were masked to treatment assignment. Placebo was produced by a pharmaceutical company, which had sacrificed their normal production in order to prepare placebo for this trial. There are also several study limitations. Considering that HbA1c mainly reflects the average blood glucose level of the previous three months, HbA1c was taken into account in this study as the primary outcome measuring efficacy. However, the duration of the study is still rather short for already established drugs which might need further long-term clinical trials to provide more information to clinicians. In addition, the study groups are Chinese adults, which might not be extended to other populations. Besides, only three points of blood samples were collected; we did not collect more detailed information of glucose change, insulin, glucagon or drug levels.

In conclusion, our study demonstrated that sitagliptin $100 \mathrm{mg}$ once daily, vildagliptin $50 \mathrm{mg}$ twice daily and saxagliptin $5 \mathrm{mg}$ once daily have similar efficacy and short-term side effects profiles in patients who were either treatment-naive or off prior anti-hyperglycemic agent therapy for at least 3 months, supporting the use of DPP-4 inhibitors in patients with newly diagnosed diabetes.

\section{Supplementary data}

This is linked to the online version of the paper at https://doi.org/10.1530/ EC-18-0523.

\section{Declaration of interest}

The pharmaceutical companies (Merck Sharp \& Dohme Italia SPA, Novartis Pharma Stein AG, AstraZeneca Pharmaceuticals LP and Qilu Pharmaceutical Co., Ltd, China) involved in the study declare that there are no conflicts of interest.

\section{Funding}

This work was funded by National Natural Science Foundation of China (No. 81570742).

\section{Author contribution statement}

The study was conceived and designed by $L$ L, J D and L S. X Z and J L performed the research and were involved in acquisition of the data. $L D$ analyzed and interpreted the data. L D and X Z drafted the manuscript. All authors approved the final version of the manuscript. $X Z$ and $L D$ contributed equally to this work and were co-first authors. L L, J D and L S worked as co-authors for correspondence.

\section{Acknowledgements}

Thanks for the support from the Pharmacy Department in Shandong Provincial Qianfoshan Hospital. This trial is registered with ClinicalTrials. gov, number, NCT01703637.

\section{References}

1 International Diabetes Federation. IDF Diabetes Atlas, 8th ed. Brussels, Belgium: IDF. (available at: http://www.diabetesatlas.org/)

2 Wang L, Gao P, Zhang M, Huang Z, Zhang D, Deng Q, Li Y, Zhao Z, Qin $X$, Jin D, et al. Prevalence and ethnic pattern of diabetes and prediabetes in China in 2013. JAMA 2017317 2515-2523. (https:// doi.org/10.1001/jama.2017.7596)

3 Edelman SV \& Polonsky WH. Type 2 diabetes in the real world: the elusive nature of glycemic control. Diabetes Care 201740 1425-1432. (https://doi.org/10.2337/dc16-1974)

4 Xu Y, Wang L, He J, Bi Y, Li M, Wang T, Wang L, Jiang Y, Dai M, Lu J, et al. Prevalence and control of diabetes in Chinese adults. JAMA 2013310 948-959. (https://doi.org/10.1001/jama.2013.168118)

5 Connelly KA, Yan AT, Leiter LA, Bhatt DL \& Verma S. Cardiovascular implications of hypoglycemia in diabetes mellitus. Circulation 2015132 2345-2350. (https://doi.org/10.1161/ CIRCULATIONAHA.115.015946)

6 American Diabetes Association. 8. Pharmacologic approaches to glycemic treatment: standards of medical care in diabetes-2018. Diabetes Care 201841 S73-S85. (https://doi.org/10.2337/dc18-S008)

7 Davies MJ, D'Alessio DA, Fradkin J, Kernan WN, Mathieu C, Mingrone G, Rossing P, Tsapas A, Wexler DJ \& Buse JB. Management of hyperglycemia in Type 2 diabetes, 2018. A consensus report by the American Diabetes Association (ADA) and the European Association for the Study of Diabetes (EASD). Diabetes Care 201841 2669-2701. (https://doi.org/10.2337/dci18-0033)

8 Scirica BM, Braunwald E, Raz I, Cavender MA, Morrow DA, Jarolim P, Udell JA, Mosenzon O, Im K, Umez-Eronini AA, et al. Heart failure, saxagliptin, and diabetes mellitus: observations from the
This work is licensed under a Creative Commons Attribution-NonCommercial-NoDerivatives 4.0 Anternationab ticense.ifica.com at 04/26/2023 09:57:38AM 
SAVOR-TIMI 53 randomized trial. Circulation 2014130 1579-1588. (https://doi.org/10.1161/CIRCULATIONAHA.114.010389)

9 Scirica BM, Bhatt DL, Braunwald E, Steg PG, Davidson J, Hirshberg B, Ohman P, Frederich R, Wiviott SD, Hoffman EB, et al. Saxagliptin and cardiovascular outcomes in patients with type 2 diabetes mellitus. New England Journal of Medicine 2013369 1317-1326. (https://doi. org/10.1056/NEJMoa1307684)

10 Munir KM \& Lamos EM. Diabetes type 2 management: what are the differences between DPP-4 inhibitors and how do you choose? Expert Opinion on Pharmacotherapy 201718 839-841. (https://doi.org/10.108 $0 / 14656566.2017 .1323878)$

11 Kim YG, Hahn S, Oh TJ, Kwak SH, Park KS \& Cho YM. Differences in the glucose-lowering efficacy of dipeptidyl peptidase-4 inhibitors between Asians and non-Asians: a systematic review and metaanalysis. Diabetologia 201356 696-708. (https://doi.org/10.1007/ s00125-012-2827-3)

12 Sjostrand M, Wei C, Cook W, Johnsson K, Pollack PS, Stahre C \& Hirshberg B. Assessment of saxagliptin efficacy: meta-analysis of 14 Phase 2 and 3 clinical trials. Diabetes Therapy 20178 587-599. (https://doi.org/10.1007/s13300-017-0261-8)

13 Bekiari E, Rizava C, Athanasiadou E, Papatheodorou K, Liakos A, Karagiannis T, Mainou M, Rika M, Boura P \& Tsapas A. Systematic review and meta-analysis of vildagliptin for treatment of type 2 diabetes. Endocrine 201652 458-480. (https://doi.org/10.1007/ s12020-015-0841-1)

14 Zhan M, Xu T, Wu F \& Tang Y. Sitagliptin in the treatment of type 2 diabetes: a meta-analysis. Journal of Evidence-Based Medicine 20125 154-165. (https://doi.org/10.1111/j.1756-5391.2012.01189.x)

15 Chu WM, Ho HE, Huang KH, Tsan YT, Liou YS, Wang YH, Lee MC $\&$ Li YC. The prescribing trend of oral antidiabetic agents for type 2 diabetes in Taiwan: an 8-year population-based study. Medicine 2017 96 e8257. (https://doi.org/10.1097/MD.0000000000008257)
16 Hampp C, Borders-Hemphill V, Moeny DG \& Wysowski DK. Use of antidiabetic drugs in the U.S., 2003-2012. Diabetes Care 201437 1367-1374. (https://doi.org/10.2337/dc13-2289)

17 The Express Scripts Lab. Drug Trend Report 2016. St Louis, MI, USA: Express Scripts. (available at: http://lab.express-scripts.com/lab/drugtrend-report)

18 Farr AM, Sheehan JJ, Curkendall SM, Smith DM, Johnston SS \& Kalsekar I. Retrospective analysis of long-term adherence to and persistence with DPP-4 inhibitors in US adults with type 2 diabetes mellitus. Advances in Therapy 201431 1287-1305. (https://doi. org/10.1007/s12325-014-0171-3)

19 Li CJ, Liu XJ, Bai L, Yu Q, Zhang QM, Yu P \& Yu DM. Efficacy and safety of vildagliptin, saxagliptin or sitagliptin as add-on therapy in Chinese patients with type 2 diabetes inadequately controlled with dual combination of traditional oral hypoglycemic agents. Diabetology and Metabolic Syndrome 20146 69. (https://doi. org/10.1186/1758-5996-6-69)

20 Tatosian DA, Guo Y, Schaeffer AK, Gaibu N, Popa S, Stoch A, Langdon RB \& Kauh EA. Dipeptidyl peptidase-4 inhibition in patients with type 2 diabetes treated with saxagliptin, sitagliptin, or vildagliptin. Diabetes Therapy 20134 431-442. (https://doi. org/10.1007/s13300-013-0045-8)

21 Lamers D, Famulla S, Wronkowitz N, Hartwig S, Lehr S, Ouwens DM, Eckardt K, Kaufman JM, Ryden M, Muller S, et al. Dipeptidyl peptidase 4 is a novel adipokine potentially linking obesity to the metabolic syndrome. Diabetes 201160 1917-1925. (https://doi. org/10.2337/db10-1707)

22 Turcot V, Tchernof A, Deshaies Y, Perusse L, Belisle A, Marceau P, Hould FS, Lebel S \& Vohl MC. Comparison of the dipeptidyl peptidase-4 gene methylation levels between severely obese subjects with and without the metabolic syndrome. Diabetology and Metabolic Syndrome 20135 4. (https://doi.org/10.1186/1758-5996-5-4)

Received in final form 26 February 2019

Accepted 1 March 2019

Accepted Preprint published online 1 March 2019 https://ec.bioscientifica.com https://doi.org/10.1530/EC-18-0523 (c) 2019 The authors Published by Bioscientifica Ltd
This work is licensed under a Creative Commons Attribution-NonCommercial-NoDerivatives 4.0 Internationab ticense ifica . com at $04 / 26 / 2023 \quad 09: 57: 38 \mathrm{Am}$ 